Case Report:

\title{
Chronic Bilateral Calcified Subdural Empyema: An Unusual Complication of a Ventriculoperitoneal Shunt
}

\author{
Abdoulaye Diop ${ }^{1^{*}}$, Mohameth Faye ${ }^{2}$, Mulumba llunga Roger ${ }^{1} \mathrm{Q}$, Momar Codé Ba $^{2} \mathrm{Q}$, Seydou Boubacar Badiane ${ }^{2} \mathrm{G}$ \\ 1. Department of Neurosurgery, Regional Hospital of Thies, Thies, Senegal \\ 2. Department of Neurosurgery, Fann Teaching Hospital, University Cheikh Anta Diop, Dakar, Senegal
}

\begin{tabular}{|c|c|}
\hline $\begin{array}{l}\text { Use your device to scan } \\
\text { and read the article online }\end{array}$ & dtation: Diop A, Faye M, Ilunga Roger M, Codé Ba M, Boubacar Badiane S. Chronic Bilateral Calcified Subdural Empyema: \\
\hline atipg & $\begin{array}{l}\text { An Unusual Complication of a Ventriculoperitoneal Shunt. Iran J Neurosurg. 2020; 6(4):225-228. http://dx.doi.org/10.32598/ } \\
\text { irjns.6.4.7 }\end{array}$ \\
\hline athos & d cl": http://dx.doi.org/10.32598/irjns.6.4.7 \\
\hline
\end{tabular}

() 0

Article info:

Received: 02 Jul 2020

Accepted: 20 Sep 2020

Available Online: 01 Oct 2020

Keywords:

Complication,

Ventriculoperitoneal shunt, Subdural empyema, Bilateral, Calcified

\section{ABSTRACT}

Background and Importance: Subduralempyema is a rarecomplication ofventriculoperitoneal shunts. The ventriculoperitoneal shunt is a common technique used in the treatment of hydrocephalus. It is often plagued with multiple complications, especially infectious ones. However, the appearance of infectious complications related to ventriculoperitoneal shunt remotely from surgery is rather unusual.

Case Presentation: Through this observation, we are reporting an unusual case of chronic bilateral calcified subdural empyema which occurred 8 years after ventriculoperitoneal shunt surgery. The child underwent a bilateral craniotomy in order to evacuate the empyema and meanwhile remove the valve. One month later, the empyema relapsed along with active hydrocephalus. Consequently, the implementation of an external ventricular shunt was performed and the child benefited from adapted antibiotic therapy. After the infection was treated, a second ventriculoperitoneal shunt valve was implemented. Afterwards, the evolution was favorable, the child retained a discrete left hemiparesis as a sequel.

Conclusion: This observation is reporting the existence of late morbidity due to the insertion of a ventriculoperitoneal valve; which shows that a regular and prolonged follow-up is necessary in children with a ventriculoperitoneal shunt valve. 


\section{Highlights}

- The ventriculoperitoneal shunt is a common procedure in neurosurgical practice due to the frequency of hydrocephalus in our context.

- This surgery can be plagued by numerous complications, particularly infectious ones, which can occur in the short, medium or long term.

- The occurrence of a late discovery empyema during a ventriculoperitoneal shunt is rare.

- In light of this clinical case, regular long-term monitoring of all patients with ventriculoperitoneal shunt valves seems necessary.

\section{Plain Language Summary}

Hydrocephalus is a frequent condition in Senegal. It is often congenital and related to consanguinity in our context but there are numerous causes for it. The ventriculoperitoneal shunt is one of the most commonly used surgical techniques in our country. This procedure is often fraught with multiple complications, especially infectious ones. We are reporting through this observation the case of a 13-year-old teenager who presented with a calcified bilateral chronic subdural empyema 8 years after a ventriculoperitoneal shunt placement. He had two surgeries with a long hospital stay. Considering this clinical case, it seems necessary to establish a regular and long-term monitoring protocol for all children living with ventriculoperitoneal shunt valves.

\section{Background and Importance}

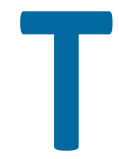

he ventriculoperitoneal shunt is a common neurosurgical procedure. Complications are considerably frequent. Reported rates of valve infections are relatively high, ranging from $3 \%$ to $15 \%$ in various studies $[1,2]$. However, the occurrence of a calcified subdural empyema as a ventriculoperitoneal shunt's complication is extremely rare [3]. Subdural empyema is a critical neurosurgical condition which may result from neurosurgical intervention, trauma, meningitis, or ear, nose, and throat (ENT) infection with reported mortality rates fluctuating from $4.4 \%$ to $24 \%$ [4]. It can cause persistent seizures, hemiparesis, or stroke. Through this observation, we are reporting an unusual case of bilateral calcified subdural empyema which occurred 8 years after a ventriculoperitoneal shunt surgery was performed in our facility with favorable outcomes.

\section{Case Presentation}

This case is about a 13-year-old teenager, who benefited from a ventriculoperitoneal shunt in $\mathbf{2 0 1 0}$ for active hydrocephalus. The post-operative course was simple, the child did not benefit from a regular follow-up afterwards. He was presented in our department with a diffuse headache which had been evolving for 2 months before his admission, associated with vomiting. The clinical examination showed an intracranial hypertension syndrome and left hemiparesis. General and neurological examinations revealed no other abnormalities. A cerebral CT scan without injection of contrast material revealed a calcified bilateral subdural empyema with a huge mass effect (Figure 1).

The empyema was surgically evacuated and the ventriculoperitoneal shunt material was removed. During the intervention, a pus sample was taken and analysis revealed a strain of Escherichia coli. The immediate post-operative course was favorable under adapted antibiotic therapy with full resolution of the signs of intracranial hypertension and a significant setback of the hemicorporeal deficit. A follow-up brain CT scan was performed 3 days after surgery, which showed that the evacuation of the empyema was satisfying with a good re-expansion of the ventricles (Figure 2).

At the clinical follow-up 1 month after surgery, the family reported a recurring headache with some episodes of vomiting. A cerebral CT scan was performed then. It showed a resurgence of subdural empyema with active hydrocephalus. The patient was subsequently taken to the operating theater and an external ventricular shunt was performed. After 3 weeks of antibiotic therapy, the valve was internalized once the Cerebro Spinal Fluid (CSF) 

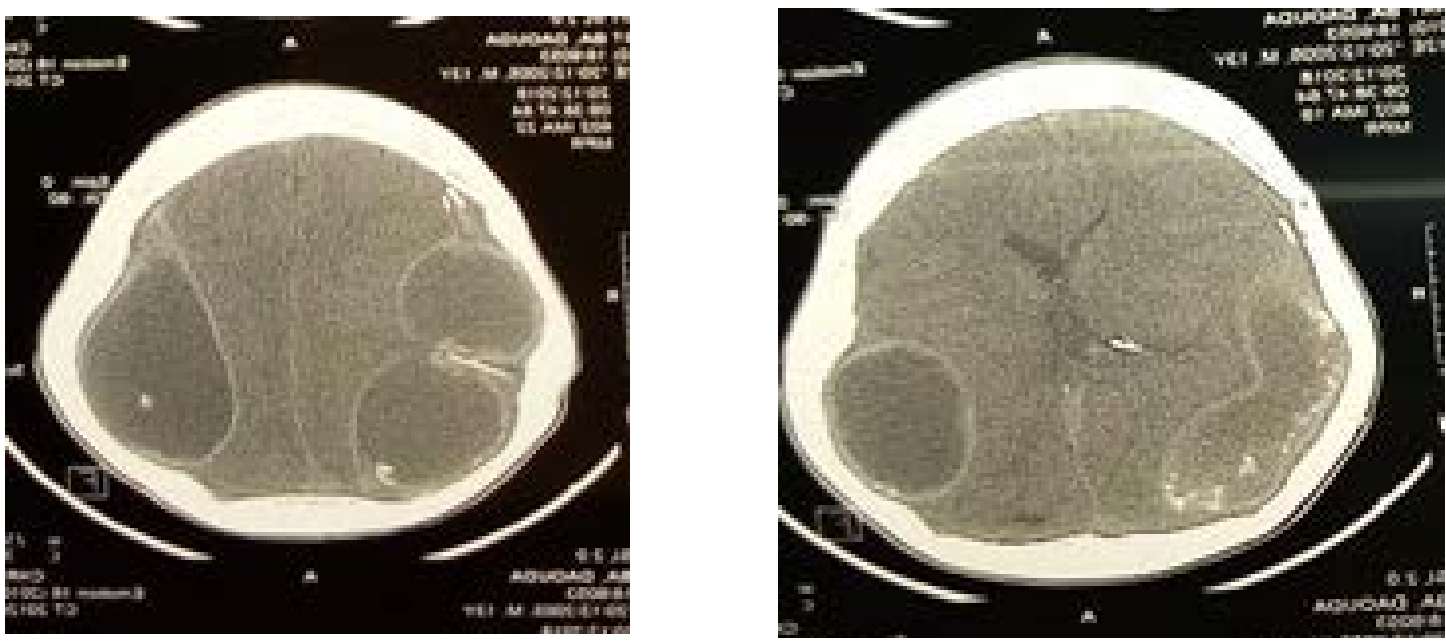

Figure 1. Non-injected pre-operative cerebral CT scan showing calcified bilateral subdural empyema with large mass effect

examination was clear. The post-operative evaluation was favorable, although the child still had a discrete left hemiparesis, which called for the initiation of a physiotherapy protocol.

\section{Discussion}

Infections are the second leading cause of ventriculoperitoneal shunt valve dysfunctions, with a reported rate fluctuating from $3 \%$ to $15 \%[1,2]$. According to many studies, the risk factors are various including young age, post-operative CSF leakage, and a lack of asepsis during surgery [2]. Valve infections usually occur between the first weeks to several months following the surgery $[1,4]$. However, a calcified subdural empyema complicating a ventriculoperitoneal shunt is extremely rare $[3,5]$. Kasliwal et al. reported a case of calcified hemispherical subdural empyema occurring 9 years after a ventriculoperitoneal shunt [3].

No case of bilateral calcified subdural empyema complicating a ventriculoperitoneal shunt has ever been reported in the literature. The spreading of the infection is variable and can reach huge proportions once the subdural space is infected [6]. The pathogenesis of subdural empyema was not elucidated in our case. The empyema is thought to be related to a subdural effusion secondarily infected either by meningitis or by contamination of the subdural space or by an infection of the operative scar $[3,7]$. The process of calcification in subdural empyema as in subdural hematoma is not well known. This process takes 3 to 12 months after pus constitution and is influenced by local factors such as blood stasis associated with a venous drainage default

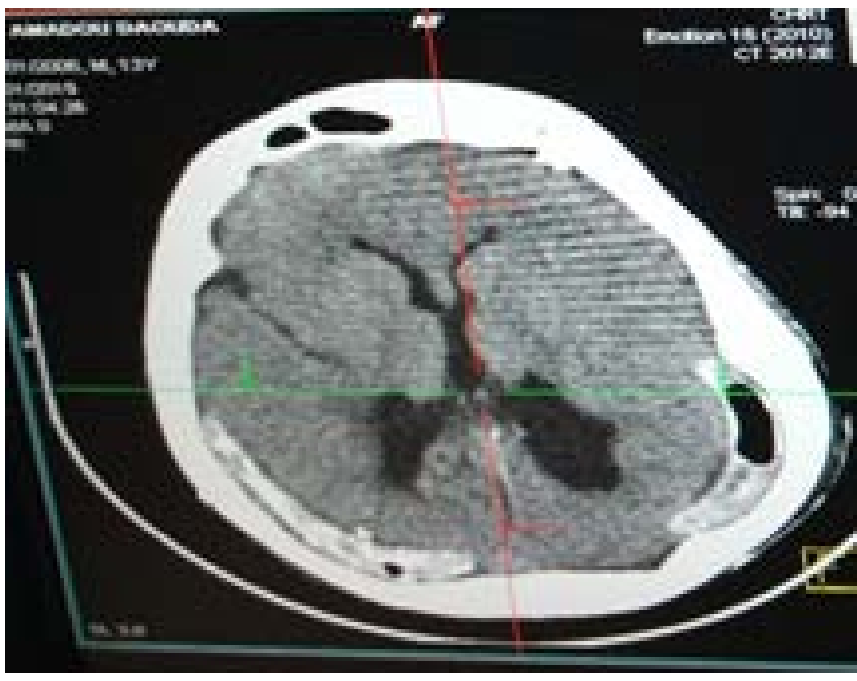

Figure 2. A follow-up brain CT scan showing a satisfactory empyema evacuation with good ventricular re-expansion on post-operative day 3 
[8]. Boyd and Merrel found that there is a metabolic predisposition to calcification, correlated to connective tissue thickness [9].

Unlike acute forms, these empyemas can remain latent for a long time. The formation of an inner membrane limits the infection and thus protects the cerebral cortex [3]. Indeed after the ossification stage, those lesions stop growing and some authors discuss the necessity of surgery $[10,11]$. In our patient's case, surgery was motivated by intracranial hypertension signs. This surgery consisted of the evacuation of the empyema with removal of the infected material concurrently. Intravenous antibiotic therapy adapted to the antibiogram was then started. This therapeutic approach is most commonly used in valve infections [12].

\section{Conclusion}

Subdural empyema is a rare but acute complication of ventriculoperitoneal shunts. However, the infection may remain latent for a long time and lead to delayed morbidity as reported in this observation which means a regular and long-term follow-up of ventriculoperitoneal shunt patients is requisite.

\section{Ethical Considerations}

\section{Compliance with ethical guidelines}

The participants were informed of the purpose of the research and its implementation stages. A written consent has been obtained from the subjects.

\section{Funding}

This research did not receive any grant from funding agencies in the public, commercial, or non-profit sectors.

Authors' contributions

All authors equally contributed to preparing this article.

Conflict of interest

The authors declared no conflict of interest. vival and etiology of failures: A seven-year institutional experience. Pediatric Neurosurgery. 2002; 36(5):248-55. [DOI:10.1159/000058428] [PMID]

[2] Kulkarni AV, Drake JM, Lamberti-Pasculli M. Cerebrospinal fluid shunt infection: A prospective study of risk factors. Journal of Neurosurgery. 2001; 94(2):195-201. [DOI:10.3171/ jns.2001.94.2.0195] [PMID]

[3] Kasliwal MK, Sinha S, Kumar R, Sharma BS. Giant hemicranial calcified subdural empyema-Unusual complication following ventriculoperitoneal shunt insertion. The Indian Journal of Pediatrics. 2009; 76(6):651-2. [DOI:10.1007/s12098009-0162-6] [PMID]

[4] Dill SR, Cobbs CG, McDonald CK. Subdural empyema: Analysis of 32 cases and review. Clinical Infectious Diseases. 1995; 20(2):372-86. [DOI:10.1093/clinids/20.2.372] [PMID]

[5] Dickerman RD, Piatt JH, Hsu F, Frank EH. Subdural empyema complicating cerebrospinal fluid shunt infection. Pediatric Neurosurgery. 1999; 30(6):310-1. [DOI:10.1159/000028816] [PMID]

[6] Kulali A, Erel C, Özyilmaz F, Sïmsek O. Giant calcified subdural empyemas. Complications. Surgical Neurology. 1994; 42(5):442-7. [DOI:10.1016/0090-3019(94)90355-7]

[7] Bakhti S, Tighilt N, Djennas M. Open drainage in chronic subdural hematomas: A prospective study of 189 cases. Iranian Journal of Neurosurgery. 2016; 2(2):15-9. [DOI:10.18869/ acadpub.irjns.2.2.15]

[8] Debois V, Lombaert A. Calcified chronic subdural hematoma. Surgical Neurology. 1980; 14(6):455-8. [Link Not Found]

[9] Boyd DA, Merrell P. Calcified subdural hematoma. The Journal of Nervous and Mental Disease. 1943; 98(6)609-17. [DOI:10.1097/00005053-194312000-00003]

[10] Watts C. The management of intracranial calcified subdural hematomas. Surgical neurology. 1976; 6(4):247-50. [PMID]

[11] Waga S, Sakakura M, Fujimoto K. Calcified subdural hematoma in the elderly. Surgical Neurology. 1979; 11(1):51-2. [PMID]

[12] Shurtleff DB, Foltz EL, Weeks RD, Loeser J. Therapy of staphylococcus epidermidis: Infections associated with cerebrospinal fluid shunts. Pediatrics. 1974; 53(1):55-62. [PMID]

\section{References}

[1] McGirt MJ, Leveque JC, Wellons JC 3rd, Villavicencio AT, Hopkins JS, Fuchs HE, et al. Cerebrospinal fluid shunt sur- 